\title{
The Learning Curves for Laser Application in Urology Procedures: Review of the Literature
}

\author{
Farzad Allameh $^{1}{ }^{\circledR}$, Mohammadreza Razzaghi ${ }^{1,2}$, Amir Reza Abedi $^{*}{ }^{\circledR}$, Mehdi Dadpour ${ }^{3}$ \\ ${ }^{1}$ Laser Application in Medical Sciences Research Center, Shahid Beheshti University of Medical Sciences, Tehran, Iran \\ ${ }^{2}$ Center of Excellence in Training Laser Application in Medicine, Shahid Beheshti University of Medical Sciences, Tehran, \\ Iran \\ ${ }^{3}$ Urology and Nephrology Research Center, Shahid Beheshti University of Medical Sciences, Tehran, Iran
}

\section{*Correspondence to Amir Reza Abedi, Department of Urology, Shohada-e-Tajrish Hospital, Shahid Beheshti University of Medical Sciences, Tehran, Iran. Email: amirezabedi@gmail.com \\ Published online December 30 2020}

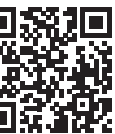

\begin{abstract}
Introduction: Currently, a laser is a popular technology in urological surgeries. The important laserrelated issue is the time when a surgeon reaches an acceptable level of safety and efficacy using laser technology.

Methods: In this review, we aimed to assess the learning curves of three types of surgeries in urology, including photoselective vaporization of the prostate (PVP), holmium laser enucleation of the prostate (HoLEP) and retrograde intra-renal surgeries (RIRSs). Here, we searched Medline, Web of Science, Google Scholar, EMBASE, and Scopus for such keywords as Urology, laser, laser vaporization, prostate, nephrolithiasis, benign prostatic hyperplasia $(\mathrm{BPH})$, and learning curve.

Results: We evaluated 14 studies about PVP, 17 about RIRS, and 29 studies about HoLEP. Also, we separately discussed the learning curves of these three kinds of surgeries in detail.

Conclusion: All the urologists, even expert surgeons, should attend a formal training course and have a skilled tutor present at their first cases.

Keywords: HoLEP; Laser; Learning curve; PVP; Surgery; Urology.
\end{abstract}

\section{Introduction}

A laser is now considered a common, effective and safe technology in almost all medical disciplines. Improvements in lasers and fiberoptics have made them perfectly appropriate to travel through routes in the human body where no hand or surgical knife has gone before. Several procedures have been established using various kinds of lasers. Today, laser applications in urology have become more prevalent. The ability of the laser to ablate prostatic tissue with minimal hemorrhage and stone fragmentation in the urinary tract has received a lot of attention. With its common use of small-diameter endoscopic devices, urology has been extremely and positively influenced by this equipment. ${ }^{1,2}$ It is very important to know that surgical experiences would affect the perioperative outcomes of the surgery. The problem with this technology, however, is the time, when a surgeon reaches the acceptable level of safety and efficacy.,

In the urology discipline, there are three important applications for lasers: I) photoselective vaporization of the prostate (PVP), II) enucleation of the prostate, and III) lithotripsy. The purpose of this review was to find evidence about learning curves in these three procedures.

\section{Materials and Methods}

This review was designed to evaluate the learning curve of three laser procedures in urology. Searches were carried out on Medline, Web of Science, Google Scholar, EMBASE, and Scopus. The keywords, including urology, laser, laser vaporization, prostate, nephrolithiasis, benign prostatic hyperplasia, and learning curve, were found.

Sixty articles were identified: 14 studies about prostate vaporization, 17 studies about lithotripsy and retrograde intra-renal surgery (RIRS), and 29 remaining ones about holmium laser enucleation of the prostate (HoLEP). Among all of these studies, the evaluation of the HoLEP learning curve was more detailed and comprehensive due to more studies in this field.

\section{Evidences}

Prostate Surgeries

Benign prostatic hyperplasia (BPH) is a common condition in elder males, resulting in obstructive lower urinary tract syndrome. Urinary frequency, urgency, difficulty starting urination, retention, infection, and renal impairment are some signs of this medical condition. Besides medical therapy, many minimally invasive techniques have been presented to treat $\mathrm{BPH}$. Changing lifestyle by signs

Please cite this article as follows: Allameh F, Razzaghi M, Abedi AR, Dadpour M. The Learning Curves for Laser Application in Urology Procedures: Review of the Literature. J Lasers Med Sci. 2020;11(suppl 1):S8-S15. doi:10.34172/jlms.2020.S2. 
and symptom relief and preserving continence status, especially in patients with higher life expectancy, are the goals of such procedures achieved through surgical training programs. Every surgeon needs to be trained and pass the learning curve period. In this work, we tried to evaluate the effect of surgeon experience on the procedure outcome. We also attempted to assess the learning curves of PVP and HoLEP by reviewing the literature..$^{1-5}$

\section{I: Photo-Selective Vaporization of the Prostate}

PVP as one of the methods to treat $\mathrm{BPH}$ has some advantages, including tissue ablation by rapid vaporization as well as rapidly hemostatic characteristics resulting in minimal intraoperative bleeding and the use of saline as an irrigating fluid resulting in less transurethral resection syndrome compared with conventional transurethral resection of the prostate (TURP). ${ }^{6,7}$ These advantages have made PVP an alternative to traditional TUR of the prostate even in a large prostate gland. Here, we intended to evaluate the learning curve and its impact on efficacy, safety, and complications.

In Seki and colleagues' study, 74 patients who had undergone PVP using a GreenLight laser at a power level of $80 \mathrm{~W}^{8}$ were evaluated in terms of the improvement rate of International Prostate Symptom Score (IPSS), quality of life score, maximum flow rate (Qmax), and postvoid residual urine after one-year follow-up. They found that all the outcomes improved after one year; however, they did not meaningfully change as the surgeon's skill increased. Items that improved with the surgeon's more familiarity were: time mandatory for tissue vaporization of the prostate adenoma and the total applied laser energy, along with the vaporized tissue weight. In their study, more experience did not decrease the total adverse incidents, including dysuria, hematuria, retention, urinary tract infection, stricture, incontinence, re-operation and blood loss. Finally, they concluded that PVP would be a good choice to treat patients with $\mathrm{BPH}$ even when the surgeon has minimal experience.

Zang et $\mathrm{al}^{9}$ evaluated the learning curve of 120 -W PVP. In their study, an endourologist, who had experienced 80 -W PVP in more than 400 cases, performed PVP on 30 consecutive patients. The pre- and post-operative volume of the prostate, lasing time, operative time, laser energy usage and laser efficiency were analyzed. Finally, they found that there were no significant differences among the patients. They concluded that endourologists highly experienced with 80-W PVP would be able to perform 120 -W PVP so that its learning curve was nearly flat for them.

In a prospective study on the learning curve for PVP using the GreenLight ${ }^{\mathrm{TM}} 180-\mathrm{W}$ XPS laser, Misrai et al ${ }^{10}$ investigated the statistics of 200 consecutive patients, who had undergone PVP. Many parameters were evaluated, including lasing time (LT), operating time (OT), LT/OT, urinary function, prostate specific antigen (PSA) serum level change, prostate volume change, hospital stay, time to Foley catheter removal, and complications based on the Clavien-Dindo score. Finally, they concluded that at least 120 procedures were required for the PVP learning curves until they met all the intra-operative factors of specialists in this surgery.

In a similar retrospective, multicentric study about the learning curve for PVP using the GreenLight ${ }^{\mathrm{TM}}$ $180-W$ XPS laser, Bastard et $\mathrm{al}^{11}$ evaluated the data from 365 consecutive patients, who had undergone PVP at three different hospitals (group 1: 152 patients, group 2: 112 patients, and group 3: 101 patients). The evaluated parameters were the operative period ( $\mathrm{min}$ ), the vaporization period $(\mathrm{min}) /$ operative period ratios, and delivered energy (Joules)/prostate volume. At last, they declared that their study, as the first work showing the outcomes found throughout the PVP learning curve, may have been influenced by patients' and urologists' characteristics. Overall, they suggested that 100 GreenLight 180-W XPS PVP trials were obligatory before touching a plateau in intraoperative parameters.

Moreover, Castellan et al ${ }^{12}$ evaluated the operating skill Influences on the safety and efficacy of PVP with a 180-W XPS GreenLight laser. In this study, they claimed that they were the first researchers who had applied learning curve analysis to see how expert and novice surgeons can be compared with each other. 291 consecutive patients with obstructive lower urinary syndrome, who had undergone PVP, were divided into two groups, 182 patients with two expert surgeons (experience of more than 130 TURP) and 109 with two novice urologists (experience of fewer than 30 TURP). Learning curves were analyzed and compared between two groups based on variations in quantitative parameters of lasing/operative period, surgery period and energy delivered. Serum PSA levels, Qmax, and IPSS improved in both groups with a higher variation in the novice group $(P<0.006)$. No significant difference was found between the two groups in relations to the total operative period; however, the lasing period/operative period ratio showed superior value for the experts compared with the novice group (0.50: IQR $0.38-0.60$ vs. 0.46: IQR $0.34-0.58$ respectively) $(P=0.201)$. A major rise in energy delivery was also attained in the novice group within the first 50 cases, whereas more than 100 procedures were required for the experts to reach LT/ OT $>60 \%$. Finally, the complication rate was found to be lower in the experts' group.

Simulators are surgical and interventional procedures without risk performed on real patients. They are both the essential part of surgical skills training and proper objective structured clinical examination tools for clinicians and residents in their clinical practice. Angulo et $\mathrm{al}^{13}$ evaluated the use of simulators for training PVP with a diode laser. They found an association between the verified efficacy parameters and diverse practice groups. They stated that repeating the process on simulators 
permits for quick learning to better face an operation.

Moreover, it is highly important and interesting to know the training recommendation of an international group of specialists on PVP for $\mathrm{BPH}$ (experience of treating more than 3500 patients at 5 centers in the United States and Europe). ${ }^{14}$ They have recommended in the beginning of practice, the first 10 patients should have prostates less than 50 grams. In addition, the first cases should not have retention, prostatitis, a large median lobe, or history of a prior prostate procedure (e.g., transurethral needle ablation or microwave therapy). An official audit on the outcome has also been suggested after performing the procedure on the first 10 patients. If there are any major complications or cases, who are unable to void spontaneously, 10 more procedures would be suggested and additional audits could be conducted on these cases. To attain an adequate capability level, almost 30-50 procedures are necessary to be performed for most trainees. A urologist should be able to use $200 \mathrm{~kJ} / \mathrm{h}$ of laser energy for operating very bulky glands. Training should be provided by instructors from an accredited training center and under the direct supervision of an expert mentor. They have not suggested the adaptation of TURP techniques because skill in the TURP surgery does not seem to be helpful to learn PVP. They have also emphasized that watching a PVP training movie alone is not adequate and even skilled urologists should join a formal training course in which a qualified tutor is present while treating their first procedures.

\section{II: Holmium Laser Enucleation of the Prostate}

The learning curve is the initial period of skill acquisition for people learning a procedure. ${ }^{15}$ The Main outcomes of holmium laser enucleation of the prostate (HoLEP) are based on operative parameters. Urologists are judged according to a main criterion, which is their skill in doing four consecutive successful procedures. A successful procedure is a procedure in which enucleation and morcellation can be done within less than 90 minutes, without conversion to TURP, with tolerable stress and difficulty. ${ }^{4}$ According to the visual analogic scale, stress and difficulty under 5/10 are satisfactory. ${ }^{16}$ Time, stress, and difficulty should be assessed for all steps of a procedure.

\section{1- Prostate Volume}

Learning HoLEP is recognized to be difficult and its learning curve depends on the prostate size. ${ }^{17}$ The efficacy of HOLEP is different in various departments and this difference can be partly explained by different prostate sizes. ${ }^{18}$ A small prostate also requires skill. Surgical plane between the surgical capsule and adenoma and also an apical plane are slightly difficult to be found in small prostates. For that reason, HoLEP is a challenging procedure in small prostates. ${ }^{19,20}$ Therefore, a moderatesize prostate is recommended for urology residents to start practicing. ${ }^{19}$ Moreover, the surgeon feels confident after $20-30$ procedures in a moderate-sized ( $50 \mathrm{~g}$ ) prostate under the supervision of an expert urologist. ${ }^{21}$ Therefore, a urologist could try smaller or larger prostates after performing at least 10-20 cases with a moderate-sized prostate. ${ }^{19,21}$ Most urologists recommend that beginner surgeons should begin with a moderate-sized prostate $(20-40 \mathrm{~g}) \cdot{ }^{17}$

\section{2- Laser}

Different laser parameters, including the used energy, the lasing period and the laser-to-prostate ratio $(\mathrm{L} / \mathrm{P}$ ratio, the volume of laser energy consumed for the removal of every gram of prostate tissue) are applied to estimate the learning curve. ${ }^{19}$ Elshal et al found that the $\mathrm{L} / \mathrm{P}$ ratio has an inverse linear relationship with the number of previously performed cases with time-to-plateau of nearly 20 procedures; later in the curve, the process is less laserassisted. Therefore, the $\mathrm{L} / \mathrm{P}$ ratio as an outcome measure is reduced during the learning curve of HoLEP. ${ }^{22}$

\section{3- Enucleation Parameters}

Different enucleation parameters have been used to evaluate the learning curve of HoLEP. Finding the surgical plane between prostate adenoma and prostate capsule requires enough skill. ${ }^{20}$ Enucleation ratio efficacy is "defined as the enucleation ratio divided by the enucleation period". Enucleation ratio efficacy is a good way to evaluate the learning curve of HoLEP. ${ }^{23}$ The efficiency of tissue enucleation, especially for a prostate $>100 \mathrm{~g}$, could tremendously improve as a surgeon's experience increases. ${ }^{24,25}$ Regarding enucleation parameters, surgical performance plateaued after the first 50-60 cases, although some urologists reported that enucleation ratio efficacy reached a plateau after 25-30 cases. $^{26}$ Enucleation ratio efficacy can remove the bias effect of prostate volume ${ }^{19,26}$; other parameters like enucleation time-energy-efficacy (ETEE = enucleated weight/enucleation time/consumed energy) could be used to evaluate enucleation skills. ${ }^{24}$ Enucleation time-energy efficacy increased after 30 procedures and plateaued between 61 to 70 cases. $^{27}$

\section{4- Morcellation Parameters}

One of the morcellation parameters is efficacy morcellation tissue/morcellation time which did not change with surgical skills in some studies and was more dependent on technology. However, some other studies showed that morcellation efficacy significantly improved with a surgeon's experience with stabilization in performance after 60 cases. ${ }^{18,23,28}$

\section{5- Postoperative Efficacy Measure}

Decrease of PSA, Enhancement in Flow Parameters and Questionnaires

The decrease of PSA after surgery was used as a predictor to evaluate the learning curve of HOLEP technique in 
3 studies, but only one study demonstrated that 4050 cases are required to reach a significant difference in this factor. ${ }^{22,24,25}$ However, the urinary flow rate or questionnaire indices did not change with increasing surgeon's skill. ${ }^{22}$

\section{6- Operative and Postoperative Safety Parameters Catheter Time and Hospital Stay}

There is controversy about the use of catheter time and the hospital stay as a way to assess the learning curve of HOLEP procedure. Some studies showed that more than 20 cases were required to reach the best result. ${ }^{16,25,29}$ But other studies did not show any correlation between this parameter and the learning curve.

\section{Perioperative Hemoglobin, Hematocrit, or Sodium Deficit} Although most studies showed that this parameter is not useful to predict the learning curve of HOLEP technique, ${ }^{23,30}$ some studies showed 25-40 cases were required to avoid disturbances in these factors. ${ }^{22,29}$

\section{Conversion of HoLEP to TURP}

The main causes for the conversion of HoLEP to TURP are either capsular perforation or uncontrolled bleeding. ${ }^{31}$ The conversion of HoLEP to TURP has been demonstrated as one of the most common parameters used as a predictor factor to assess the learning curve. ${ }^{19}$ The conversion of HoLEP to TURP decreased with increasing surgical skill (occurred in $30 \%$ of cases in the first 30 surgeries, but plummeted to $10 \%$ after 60 cases). ${ }^{32}$ The conversion of HoLEP to TURP may indirectly demonstrate the absence of a surgeon's confidence. This was reported to occur most commonly at the first 20-30 cases. ${ }^{19}$

\section{Retreatment}

The recurrence of prostate hyperplasia was seen in $4.2 \%$ of cases after HOLEP. ${ }^{33}$ The re-treatment rate in the first 50 cases was higher. ${ }^{21}$ After 50 cases, re-treatment rates significantly dropped. ${ }^{19}$

\section{Complications}

Soto-Mesa et al reported that postoperative complications 30 days after surgery did not change with increasing surgical skill. ${ }^{34}$ However, some studies showed that the number of adverse events was lower following the first 80 cases. $^{26}$

\section{Incontinence}

Persistent stress urinary incontinence more than 6 months was seen more in the first 50 cases. ${ }^{29}$ In terms of urinary incontinence, the time interval between cases and case density at the beginning of the learning curve are also important. ${ }^{29,35}$ Other factors which have effects on stress UI are the prostate size and blood loss during surgery. ${ }^{31}$ At least 20 cases are required to avoid urinary incontinence. ${ }^{28}$
De Novo Urinary Retention

De novo urinary retention is related to over-distention of the bladder throughout morcellation or myogenic damage to the detrusor muscle. ${ }^{36}$ Therefore, it is not useful to predict the learning curve of HOLEP. ${ }^{36}$

\section{Trifecta - Pentafecta}

"Trifecta has been defined as HOLEP procedure done within 90 minutes without any change to TURP, pentafecta has been reached when patients meet the trifecta criteria without postoperative complications or stress urinary incontinence". ${ }^{19}$ The learning curve has been reached when the urologist could perform four consecutive HOLEP procedures and reach the trifecta or pentafecta criteria. For HoLEP, this could happen after the 22nd and 40th surgery for trifecta and pentafecta criteria respectively. ${ }^{37}$ Training with a simulator could decrease the learning curve of HOLEP. ${ }^{38}$

\section{III: Laser Lithotripsy}

The treatment modalities of nephrolithiasis have been changed over the last two decades. Today, percutaneous nephrolithotomy (PCNL), laparoscopic pyelolithotomy, and retrograde intrarenal surgery (RIRS) are the gold standard surgical methods for kidney stone removal depending on the dimensions and site of the stones and the urologist's experience. RIRS can be used to treat intrarenal stones smaller than $3 \mathrm{~cm}$ depending on the expertise level of the surgeon, with a lower risk of kidney injury and hemorrhage in comparison with PCNL. ${ }^{39-}$ ${ }^{43}$ Although burden, location, and patients' body mass index are very important in PCNL, surgeons' skill is another determinant of the outcomes of kidney stone surgery in RIRS. To decrease operation time and quickly achieve the stone-free rate and better results with fewer complications, more training is mandatory ${ }^{44-46}$ However, there are a limited number of studies in this area. Here, we discussed a few studies about identifying learning curves for operative time, fragmentation efficacy, complication rates, and pediatrics RIRS.

In a retrospective study, Berardinelli et $\mathrm{al}^{47}$ evaluated 381 patients, who had experienced RIRS because of kidney stones, in two groups: group 1 were operated by surgeons with endo-urological experience less than 100 procedures, while group 2 were operated by surgeons with endo-urological experience more than 400 procedures. At last, they found a higher stone-free rate $(P=0.082)$, a less complication rate $(P=0.001)$, a shorter operative period $(P=0.001)$ in group 2. They concluded that surgeons' experience would affect the outcomes of RIRS.

In addition, sheath placement, stone treatment, and double J stent insertion are important steps of RIRS. da Cruz et $\mathrm{al}^{48}$ assessed the operation time of these steps and found that after 60 cases, there would be no improvement in doing the steps of surgery. Besides these variables, they also performed qualitative analysis encompassing five 
variables: tissue management, bimanual dexterity, depth perception, autonomy, and efficiency. They found that all the qualitative variables had significant differences between groups 1 (1st to 20th cases), 2 (21st through 40 th) and 3 (from the 41 st to the 60th) $(P<0.001)$ except between groups 3 and 4 (from the 61 st to the 80 th). They concluded that all the variables did not improve after performing RIRS on 60 cases.

In an experience about performing 100 consecutive RIRS, Cho et $\mathrm{al}^{49}$ introduced fragmentation efficacy calculated by the formula of removed stone volume $\left(\mathrm{mm}^{3}\right)$ divided by operative time ( $\left.\mathrm{min}\right)$, as a proper parameter to evaluate RIRS competency. By using fragmentation efficacy, the question of "How fast can a given urologist complete RIRS?” could be answered. In their study, the fragmentation efficacy touched a plateau between the 40th and 60th patients. Further evaluations showed that the curve tended to be flat until the 25th case and then revealed an increasing trend followed by a decline until the 56th case (the change point). In Cho and colleagues' study, the stone-free rate was also similar in the first 10 cases and the final 10 cases. There was no complication of Clavien classification grade 3, sepsis, and ureteral stricture in 6- to 12-month follow-up. They also indicated that the proper level of fragmentation efficacy was a minimum of $25 \mathrm{~mL} / \mathrm{min}$ equivalent to a 60 -minute operation for a calculated stone volume of $20 \mathrm{~mm} \times 15$ $\mathrm{mm} \times 10 \mathrm{~mm} \times 0.523$.

One of the main benefits of flexible ureteroscopy is that all part of the urinary tract is available, but it would be accompanied by some complications. Ureteroscopy is one of the most common reasons for ureteral damage. ${ }^{50}$ Mucosal injury, ureteral perforation, ureteral avulsion, urinoma formation, bleeding, and ureteral stricture are some possible complications during flexible ureteroscopy. Along with many studies of flexible ureteroscopy complications, Komori et $\mathrm{al}^{51}$ evaluated these complications during the learning curve time. The patients were assigned to 4 groups based on the physician's skill: group 1 RIRS was operated by less experienced surgeons ( $n=35)$, group 2 for the 36 th to 85 th cases $(n=50$, training at a high-volume hospital), group 3 included the next 50 cases (86th -135th), and group 4 covered cases 136th to 219th. The mean operation period decreased from group 1 to group $4(P<0.05)$. On the other hand, the mean stone-free rate increased from group 1 to group 4 ( $P$ $<0.05)$. The rate of complication, based on Clavien score (Clavien 2-4), was 3.2\%, 8.3\%, 10\%, and $13.6 \%$ in groups 4 , 3,2 and 1 respectively $(P=0.068)$. Finally, they concluded that to perform safe and successful surgery with fewer complications, it is mandatory to have experience of 100 cases at a high-volume center.

Compared to adult patients, urolithiasis is less common in children; however, children may be subject to more morbidity. ${ }^{52}$ Today, there is an increasing use of RIRS compared to PCNL and mini-PCNL in the treatment of pediatrics urolithiasis due to some benefits, including lower bleeding and radiation exposure and a shorter hospital stay. ${ }^{53,54}$ Regarding pediatrics, there is a concern whether or not an endo-urologist, who has had enough experience in an adult procedure, would be also able to perform this kind of surgery on children. In other words, do adult endo-urologists require a specific learning curve for children? Sforza et $\mathrm{al}^{55}$ evaluated the safety and feasibility of performing RIRS in children by endourologists, who are expert in adult surgery but without experience in pediatrics. They compared 30 consecutive patients (15 adults and 15 children) with similar perioperative data, including stone location and size. The patients had also undergone RIRS by the same surgeon with experience of more than 500 RIRS in adults but no experience with children. They did not find any difference in complications between the two groups, including fever and hematuria, the extent of hospitalization, and the stone-free rate. Finally, they concluded that an adult endourologist does not need a specific and separate learning curve for children and the results of pediatric cases would be comparable to adults.

In this study, we reviewed available related literature on the learning curves for three common urologic procedures by using laser technology. With respect to HoLEP, we discussed subheading categories in detail due to more published studies in this field. However, regarding PVP and RIRS, there were only a few studies. Therefore, there seemed to be urgent need for more similar studies to discuss the parameters of learning curves in PVP and RIRS in more detail and comprehensively. We also emphasized that viewing surgeries performed by lasers in a urology video is not sufficient and even skilled surgeons should join an official training course, in which a qualified guide exists at initial surgeries. To evaluate the learning curve of lasers in urology procedures, we recommend that further studies also consider the surgeon's experiences in other surgical methods.

\section{Conclusion}

In this review, we appraised the literature about the learning curve of laser applications in three common urology surgeries including RIRS, PVP and HoLEP. We could discuss HoLEP surgery in more detail rather than the others because of more published studies.

\section{Ethical Considerations}

Not applicable.

\section{Conflict of Interests}

The authors declare that they have no conflict of interest.

\section{References}

1. Abedi A, Razzaghi MR, Rahavian A, Hazrati E, Aliakbari F, Vahedisoraki V, et al. Is Holmium laser enucleation of 
the prostate a good surgical alternative in benign prostatic hyperplasia management? A review article. J Lasers Med Sci. 2020;11(2):197-203. doi: 10.34172/jlms.2020.33.

2. Allameh F, Basiri A, Razzaghi M, Abedi AR, Fallah-Karkan $\mathrm{M}$, Ghiasy S, et al. Clinical efficacy of transurethral resection of the prostate combined with oral anticholinergics or botulinum toxin - A injection to treat benign prostatic hyperplasia with overactive bladder: A case-control study. Clin Pharmacol. 2020;12:75-81. doi: 10.2147/CPAA. S256051.

3. Saredi G, Pirola GM, Pacchetti A, Lovisolo JA, Borroni G, Sembenini F, et al. Evaluation of the learning curve for thulium laser enucleation of the prostate with the aid of a simulator tool but without tutoring: comparison of two surgeons with different levels of endoscopic experience. BMC Urol. 2015;15:49. doi: 10.1186/s12894-015-0045-2.

4. Enikeev D, Glybochko P, Rapoport L, Gahan J, Gazimiev $\mathrm{M}$, Spivak L, et al. A randomized trial comparing the learning curve of 3 endoscopic enucleation techniques (HoLEP, ThuFLEP, and MEP) for BPH using mentoring approach-initial results. Urology. 2018;121:51-7. doi: 10.1016/j.urology.2018.06.045.

5. Simforoosh N, Dadpour M, Mousapour P, Honarkar Ramezani M. Improving early urinary continence recovery after radical prostatectomy by applying a sutureless technique for maximal preservation of the intrapelvic urethra: A 17-year single-surgeon experience. Urologia. 2020;87(4):178-84. doi: 10.1177/0391560320925570.

6. Malek RS. Photoselective KTP laser vaporization of obstructive BPH (PVP). In: Baba S, Ono Y, editors. Interventional management of urological diseases. Recent advances in endourology. Vol. 8. Tokyo: Springer; 2006.

7. Rassweiler J, Teber D, Kuntz R, Hofmann R. Complications of transurethral resection of the prostate (TURP)-incidence, management, and prevention. Eur Urol. 2006;50(5):969-80. doi: 10.1016/j.eururo.2005.12.042.

8. Seki N, Nomura H, Yamaguchi A, Naito S. Evaluation of the learning curve for photoselective vaporization of the prostate over the course of 74 cases. J Endourol. 2008;22(8):1731-5. doi: 10.1089/end.2007.0368.

9. Zang Y, Shan Y, Xue B, Yang D, Sun C, Gao J. UP-3.082: The learning curve of $120-\mathrm{W}$ photoselective vaporization of the prostate for benign prostatic hyperplasia. Urology. 2009;74(4):S319-S20. doi: 10.1016/j.urology.2009.07.086.

10. Misrai V, Faron M, Guillotreau J, Bruguiere E, Bordier $\mathrm{B}$, Shariat SF, et al. Assessment of the learning curves for photoselective vaporization of the prostate using GreenLight $^{\text {tix }}$ 180-Watt-XPS laser therapy: defining the intra-operative parameters within a prospective cohort. World J Urol. 2014;32(2):539-44. doi: 10.1007/s00345-0131163-7.

11. Bastard C, Misrai V, Roupret M, Zorn K, Hueber PA, Peyronnet B. MP13-09 assessment of the learning curves for prostate photoselective vaporization using GreenLight ${ }^{\mathrm{TM}}$ 180-Watt-XPS laser therapy: A multicentric study. J Urol. 2017;197(4S):e156-e157. doi: 10.1016/j.juro.2017.02.452.

12. Castellan P, Marchioni M, Rizzoli A, Di Nicola M, Porfilio I, Ghahhari J, et al. The surgical experience influences the safety and efficacy of photovaporization of prostate with 180-W XPS GreenLight laser: Comparison between novices vs expert surgeons learning curves. J Endourol. 2018;32(11):1071-7. doi: 10.1089/end.2018.0437.

13. Angulo JC, Arance I, Garcia-Tello A, Las Heras MM, Andres $\mathrm{G}$, Gimbernat $\mathrm{H}$, et al. Virtual reality simulator for training on photoselective vaporization of the prostate with $980 \mathrm{~nm}$ diode laser and learning curve of the technique. Actas Urol Esp. 2014;38(7):451-8. doi: 10.1016/j.acuroe.2014.06.003.

14. Gomez Sancha F, Bachmann A, Choi BB, Tabatabaei S, Muir GH. Photoselective vaporization of the prostate (GreenLight PV): lessons learnt after 3500 procedures. Prostate Cancer Prostatic Dis. 2007;10(4):316-22. doi: 10.1038/sj.pcan.4500989.

15. Subramonian K, Muir G. The 'learning curve' in surgery: what is it, how do we measure it and can we influence it? BJU Int. 2004;93(9):1173-4. doi: 10.1111/j.1464410X.2004.04891.x.

16. Robert G, Cornu JN, Fourmarier M, Saussine C, Descazeaud A, Azzouzi AR, et al. Multicentre prospective evaluation of the learning curve of holmium laser enucleation of the prostate (HoLEP). BJU Int. 2016;117(3):495-9. doi: 10.1111/bju.13124.

17. Haraguchi T, Takenaka A, Yamazaki T, Nakano Y, Miyake H, Tanaka K, et al. The relationship between the reproducibility of holmium laser enucleation of the prostate and prostate size over the learning curve. Prostate Cancer Prostatic Dis. 2009;12(3):281-4. doi: 10.1038/pcan.2009.16.

18. Shah HN, Mahajan AP, Sodha HS, Hegde S, Mohile PD, Bansal MB. Prospective evaluation of the learning curve for holmium laser enucleation of the prostate. J Urol. 2007;177(4):1468-74. doi: 10.1016/j.juro.2006.11.091.

19. Kampantais S, Dimopoulos P, Tasleem A, Acher P, Gordon $\mathrm{K}$, Young A. Assessing the learning curve of Holmium laser enucleation of prostate (HoLEP). A systematic review. Urology. 2018;120:9-22. doi: 10.1016/j.urology.2018.06.012.

20. Kim M, Lee HE, Oh SJ. Technical aspects of holmium laser enucleation of the prostate for benign prostatic hyperplasia. Korean J Urol. 2013;54(9):570-9. doi: 10.4111/ kju.2013.54.9.570.

21. Elzayat EA, Khalaf I, Elgallad M, Hosny H, Elhilali M. Holmium laser enucleation of prostate in patients with prostate size $\leq 60 \mathrm{~cm}^{3}$. Urology. 2009;73(1):95-9. doi: 10.1016/j.urology.2008.06.049.

22. Elshal AM, Nabeeh H, Eldemerdash Y, Mekkawy R, Laymon M, El-Assmy A, et al. Prospective assessment of learning curve of Holmium Laser enucleation of the prostate for treatment of benign prostatic hyperplasia using a multidimensional approach. J Urol. 2017;197(4):1099107. doi: 10.1016/j.juro.2016.11.001.

23. Jeong CW, Oh JK, Cho MC, Bae JB, Oh SJ. Enucleation ratio efficacy might be a better predictor to assess learning curve of holmium laser enucleation of the prostate. Int Braz J Urol. 2012;38(3):362-72. doi: 10.1590/s167755382012000300009.

24. Seki N, Mochida O, Kinukawa N, Sagiyama K, Naito S. Holmium laser enucleation for prostatic adenoma: analysis of learning curve over the course of 70 consecutive cases. J Urol. 2003;170(5):1847-50. doi: 10.1097/01. ju.0000092035.16351.9d.

25. Hwang JC, Park SM, Lee JB. Holmium laser enucleation of the prostate for benign prostatic hyperplasia: effectiveness, 
safety, and overcoming of the learning curve. Korean J Urol. 2010;51(9):619-24. doi: 10.4111/kju.2010.51.9.619.

26. Brunckhorst O, Ahmed K, Nehikhare O, Marra G, Challacombe B, Popert R. Evaluation of the learning curve for Holmium laser enucleation of the prostate using multiple outcome measures. Urology. 2015;86(4):824-9. doi: 10.1016/j.urology.2015.07.021.

27. Kim KH, Kim KT, Oh JK, Chung KJ, Yoon SJ, Jung H, et al. Enucleated weight/enucleation Time, Is it appropriate for estimating enucleation skills for Holmium laser enucleation of the prostate? A consideration of energy consumption. World J Mens Health. 2018;36(1):79-86. doi: 10.5534/wjmh.17039.

28. Shigemura K, Yamamichi F, Kitagawa K, Yamashita M, Oka $\mathrm{Y}$, Tanaka $\mathrm{H}$, et al. Does surgeon experience affect operative time, adverse events and continence outcomes in Holmium laser enucleation of the prostate? A review of more than 1,000 cases. J Urol. 2017;198(3):663-70. doi: 10.1016/j. juro.2017.04.087.

29. Placer J, Gelabert-Mas A, Vallmanya F, Manresa JM, Menendez V, Cortadellas R, et al. Holmium laser enucleation of prostate: outcome and complications of self-taught learning curve. Urology. 2009;73(5):1042-8. doi: 10.1016/j.urology.2008.12.052.

30. El-Hakim A, Elhilali MM. Holmium laser enucleation of the prostate can be taught: the first learning experience. BJU Int . 2002;90(9):863-9. doi: 10.1046/j.1464-410x.2002.03071.x.

31. Kobayashi S, Yano M, Nakayama T, Kitahara S. Predictive risk factors of postoperative urinary incontinence following holmium laser enucleation of the prostate during the initial learning period. Int Braz J Urol. 2016;42(4):740-6. doi: 10.1590/S1677-5538.IBJU.2015.0477.

32. Bae J, Oh SJ, Paick JS. The learning curve for holmium laser enucleation of the prostate: a single-center experience. Korean J Urol. 2010;51(10):688-93. doi: 10.4111/ kju.2010.51.10.688.

33. Jeon BJ, Chung H, Bae JH, Jung H, Lee JG, Choi H. Analysis of present status for surgery of benign prostatic hyperplasia in Korea using nationwide healthcare system data. Int Neurourol J. 2019;23(1):22-9. doi: 10.5213/inj.1836198.099.

34. Soto-Mesa D, Amorin-Diaz M, Perez-Arviza L, FernandezPello Montes S, Martin-Huescar A. Holmium laser enucleation of the prostate and retropubic prostatic adenomectomy: morbidity analysis and anesthesia considerations. Actas Urol Esp. 2015;39(9):535-45. doi: 10.1016/j.acuroe.2015.09.007

35. Lerner LB, Tyson MD, Mendoza PJ. Stress incontinence during the learning curve of holmium laser enucleation of the prostate. J Endourol. 2010;24(10):1655-8. doi: 10.1089/ end.2010.0021.

36. Kim SH, Yoo C, Choo M, Paick JS, Oh SJ. Factors affecting de novo urinary retention after Holmium laser enucleation of the prostate. PloS One. 2014;9(1):e84938. doi: 10.1371/ journal.pone.0084938.

37. Peyronnet B, Robert G, Comat V, Roupret M, GomezSancha F, Cornu JN, et al. Learning curves and perioperative outcomes after endoscopic enucleation of the prostate: a comparison between GreenLight 532-nm and holmium lasers. World J Urol. 2017;35:973-83. doi: 10.1007/s00345016-1957-5.
38. Tan SS, Sarker SK. Simulation in surgery: a review. Scott Med J. 2011;56(2):104-9. doi: 10.1258/smj.2011.011098.

39. Abedi AR, Razzaghi MR, Allameh F, Aliakbari F, FallahKarkan M, Ranjbar A. Pneumatic lithotripsy versus laser lithotripsy for ureteral Stones. J Lasers Med Sci. 2018;9(4):233-6. doi: 10.15171/jlms.2018.42.

40. Allameh F, Razzaghi M, Fallah-Karkan M, Hosseini B, Tayyebi Azar A, Ranjbar A, et al. Comparison of stone retrieval basket, stone cone and Holmium laser: Which one is better in retropulsion and stone-free status for patients with upper ureteral calculi? J Lasers Med Sci. 2019;10(3):179-84. doi: 10.15171/jlms.2019.28.

41. Simforoosh N, Radfar MH, Valipour R, Dadpour M, Kashi AH. Laparoscopic Pyelolithotomy for the Management of Large Renal Stones with Intrarenal Pelvis Anatomy. Urol J. 2020;16(7). doi:10.22037/uj.v0i0.5576.

42. Radfar $\mathrm{MH}$, Dadpour $\mathrm{M}$, Simforoosh $\mathrm{N}$, Basiri A, Nouralizadeh A, Shakiba B, et al. Laparoscopic pyelolithotomy in patients with previous ipsilateral renal stone surgery. Urologia. 2019:391560319890993. doi: 10.1177/0391560319890993.

43. Akman T, Binbay M, Ugurlu M, Kaba M, Akcay M, Yazici O, et al. Outcomes of retrograde intrarenal surgery compared with percutaneous nephrolithotomy in elderly patients with moderate-size kidney stones: a matched-pair analysis. J Endourol. 2012;26(6):625-9. doi: 10.1089/end.2011.0526.

44. Skolarikos A, Gravas S, Laguna MP, Traxer O, Preminger GM, de la Rosette J. Training in ureteroscopy: a critical appraisal of the literature. BJU Int. 2011;108(6):798-805. doi: 10.1111/j.1464-410X.2011.10337.x.

45. Weinberg JJ, Ansong K, Smith AD. Complications of ureteroscopy in relation to experience: report of survey and author experience. J Urol. 1987;137(3):384-5. doi: 10.1016/ s0022-5347(17)44040-7.

46. Razzaghi MR, Fallah Karkan M, Ghiasy S, Javanmard B. Laser application in iran urology: A narrative review. J Lasers Med Sci. 2018;9(1):1-6. doi: 10.15171/jlms.2018.01.

47. Berardinelli F, Cindolo L, De Francesco P, Proietti S, Hennessey D, Dalpiaz O, et al. The surgical experience influences the safety of retrograde intrarenal surgery for kidney stones: a propensity score analysis. Urolithiasis. 2017;45(4):387-92. doi: 10.1007/s00240-016-0919-0.

48. da Cruz JA, Thiago C, de Queiros Barros U, de la Roca RL , Lima JP, Di Migueli R. MP33-17 the learning curve for retrograde intra-renal surgery (RIRS): How many cases are necessary? J Urol. 2016;195(4S):e443. doi: 10.1016/j. juro.2016.02.1374.

49. Cho SY, Choo MS, Jung JH, Jeong CW, Oh S, Lee SB, et al. Cumulative sum analysis for experiences of a singlesession retrograde intrarenal stone surgery and analysis of predictors for stone-free status. PloS One. 2014;9(1):e84878. doi: 10.1371/journal.pone.0084878.

50. Brooke Johnson D, Pearle MS. Complications of ureteroscopy. Urol Clin North Am. 2004;31(1):157-71. doi: 10.1016/S0094-0143(03)00089-2.

51. Komori M, Izaki H, Daizumoto K, Tsuda M, Kusuhara $\mathrm{Y}$, Mori $\mathrm{H}$, et al. Complications of flexible ureteroscopic treatment for renal and ureteral calculi during the learning curve. Urol Int. 2015;95(1):26-32. doi: 10.1159/000368617.

52. Clayton DB, Pope JC. The increasing pediatric stone 
disease problem. Ther Adv Urol. 2011;3(1):3-12. doi: $10.1177 / 1756287211400491$.

53. Suliman A, Burki T, Garriboli M, Glass J, Taghizadeh A. Flexible ureterorenoscopy to treat upper urinary tract stones in children. Urolithiasis. 2020;48(1):57-61. doi: 10.1007/s00240-018-1083-5.

54. Bas O, Dede O, Aydogmus Y, Utangac M, Yikilmaz TN, Damar E, et al. Comparison of retrograde intrarenal surgery and micro-percutaneous nephrolithotomy in moderately sized pediatric kidney stones. J Endourol. 2016;30(7):76570. doi: 10.1089/end.2016.0043.

55. Sforza S, Tuccio A, Grosso AA, Crisci A, Cini C, Masieri L. Could surgical experience of adult endourologist overcome the learning curve of retrograde intrarenal surgery in children? Urolithiasis. 2020;48(5):459-64. doi: 10.1007/ s00240-019-01161-x. 